\title{
Rédiger un protocole douleur : quelle méthode atraumatique pour l'urgentiste?
}

\author{
Writing a Pain Protocol for the Emergency Department: Which Method for an atraumatic \\ Experience?
}

\author{
V.-E. Lvovschi \\ Reçu le 8 octobre 2017; accepté le 20 janvier 2018 \\ (C) SFMU et Lavoisier SAS 2018
}

Résumé Les protocoles " antalgie » sont des outils incontournables pour tous les services d'urgence dans le cadre de l'amélioration de la prise en charge de la douleur. Suivre les recommandations d'experts ne peut suffire. Un protocole doit proposer des conduites à tenir ciblées sur des situations définies, validées scientifiquement, adaptées au contexte du service et au personnel concerné, dans un cadre réglementaire et légal précis. Mais l'élaboration d'un protocole peut s'avérer une expérience pénible pour l'urgentiste, même si c'est une des missions extracliniques essentielles à la professionnalisation du métier. Pour le non-algologue, sa conception et sa rédaction peuvent être perçues comme fastidieuses et non prioritaires, mais cette inertie retarde dangereusement la mise à jour de nos stratégies thérapeutiques globales, en particulier en traumatologie. L'optimisation de cette tâche et un gain de temps sont pourtant possibles grâce à un guide méthodologique, des modèles, des documents types, tous accessibles, mais parfois mal référencés : l'expert pourra ainsi consacrer son temps au contenu et à l'adhésion des équipes plutôt qu'à la forme et, in fine, au patient. Une « protocolisation » de l'élaboration de nos protocoles est sans doute le moyen de les réaliser et de les mettre à jour plus efficacement, plus vite et sans douleur. Se doter d'un protocole d'antalgie est en fait une opportunité pour les équipes davantage qu'une contrainte, à condition de proposer une méthodologie adaptée à la culture de la médecine d'urgence, transversale, accessible, priorisée.

Mots clés Douleur · Urgences · Protocoles de soins · Méthodologie

V.-E. Lvovschi $(\bowtie)$

Service d'accueil des urgences, centre hospitalier universitaire Charles-Nicolle, 1, rue de Germont, F-76000 Rouen, France

e-mail : virginie.lvovschi@chu rouen.fr

\begin{abstract}
Analgesia protocols are essential tools for all emergency departments as part of the improvement of pain management. Follow Expert Guidelines cannot be enough. A protocol must propose procedures targeted on defined clinical situations, scientifically validated, adapted to the context of the emergency department and to the staff members concerned, within a precise regulatory and legal framework. But developing a protocol may be a painful experience for the emergency physician, even if it is one of the extra-clinical missions essential for our specialty's professionalization. For the non-algologist, its conception and writing can be perceived as tedious and non-priority, but this inertia dangerously delays the updating of our therapeutic strategies, especially in traumatology. Optimizing this task and saving time is however possible thanks to methodological guides, models and templates, all accessible but sometimes poorly referenced: The expert can thus devote his time to the content and the adhesion of the teams rather than to formatting protocols and, in fine, to the patient. A "protocolization" of the development of our protocols is probably the way to realize them and to update them more efficiently, faster and painlessly. Having an "analgesia protocol" is in fact an opportunity for the teams rather than a constraint, provided that we propose a methodology adapted to the culture of emergency medicine, transversal, accessible, prioritized.
\end{abstract}

Keywords Pain · Emergency $\cdot$ Treatment protocols · Methodology

\section{Introduction}

De nouvelles stratégies de prise en charge de la douleur aiguë aux urgences sont apparues ces dernières années, en particulier en traumatologie [1-4]. L'urgentiste, comme tout spécialiste, doit tenir compte de l'évolution des connaissances pour adapter et mettre à jour sa pratique quotidienne et 
celle des équipes soignantes. Ce changement est simple quand il s'agit de prescriptions nominatives, mais nécessite de créer ou de mettre à jour toute une série de procédures quand l'antalgie est protocolisée. Le mot "protocole " répond à de multiples définitions, mais selon son étymologie (du grec prôtokollon — « ce qui est collé en premier »), il désignait le premier morceau d'un rouleau de papyrus, portant un sceau [5], le protocole est donc un document officiel, constituant le « procès-verbal d'une réunion », le « texte d'un engagement ». Il s'agit d'un « ensemble de règles, de questions, etc., définissant une opération complexe ». Appliqué à la médecine, un protocole officialise l'application de recommandations, précise les étapes d'une prise en charge, impose des consignes [6]. Le protocole est donc synonyme de contrainte et peut paraître limiter le champ de prescription de l'urgentiste, voire sa liberté d'action, sans contrepartie tangible. Ces protocoles sont pourtant particulièrement utiles dans le cas de la douleur aiguë aux urgences. Une prise en charge rapide, codifiée, déléguée, sécurisée, permet aux équipes d'accueil de soulager la plainte souvent prioritaire du patient, et au médecin de se polariser sur le diagnostic et le traitement étiologique. Mais leur rédaction peut paraître complexe, trop institutionnelle, longue et loin des priorités de l'urgentiste. Ce ressenti est source d'inertie au changement au moment de mettre à jour nos stratégies thérapeutiques. Pourtant, en douleur aiguë, les protocoles sont en parfaite adéquation avec nos besoins, nos objectifs, et notre culture. L'élaboration d'un protocole est parfois une expérience quasi traumatique pour l'urgentiste, qui refuse souvent d'en élaborer un deuxième quelques années après. Pouvons-nous faire mieux, plus sereinement et plus vite ? La mise en place d'un protocole a en fait de multiples effets pléiotropes positifs pour les urgentistes eux-mêmes et pour l'ensemble des membres d'un service d'urgence, une fois les premières appréhensions vaincues. Cet article aborde quelques grandes questions concernant l'environnement médical et réglementaire des protocoles douleur aiguë, et propose quelques pistes méthodologiques et pratiques pour accomplir cette tâche importante... sans douleur.

\section{Protocole : contraintes et opportunités}

\section{Quelles sont les contraintes de rédaction?}

Les recommandations professionnelles sont définies par la Haute Autorité de santé comme «des propositions développées selon une méthode explicite pour aider le praticien et le patient à rechercher les soins les plus appropriés dans des circonstances cliniques données ». Les recommandations ayant une vocation nationale et générale, elles ne proposent pas de protocole, mais un algorithme décisionnel indiquant les possibilités thérapeutiques dans une approche multimo- dale (Fig. 1) [7]. La procédure est «la manière spécifiée d'accomplir une activité » (norme ISO $9000 ; 2000$ ). La procédure décrit la manière d'accomplir une action : domaine d'application et objet de l'activité, intervenant, manière de faire, matériel utilisé et façon dont est maîtrisée et enregistrée l'action. La fiche technique est la «description méthodique et chronologique des opérations successives à effectuer pour la réalisation d'une tâche, d'un acte de soins. Elle doit être validée, remise à jour périodiquement et lors de chaque évolution technique, scientifique, législative " (BO 92-13 bis, ministère de la Santé). Le protocole est un « descriptif de techniques à appliquer et/ou de consignes à observer " (ANAES, Agence nationale d'accréditation et d'évaluation en santé). Il s'agit donc d'un guide d'application des procédures, centré sur une cible (par exemple une population de patients), présenté sous forme synthétique. Il intègre à la fois les recommandations nationales et leur adaptation sous forme de procédures et de fiches techniques. La traduction des recommandations dans la pratique nécessite des protocoles de soins spécifiques adaptés au contexte local (ressources, ciblage de populations ou des gestes spécifiques, mise en cohérence avec les autres protocoles de l'établissement...) (Tableau 1). On observe ainsi une grande diversité des protocoles en France et en Europe pour une même pathologie, malgré les recommandations d'experts $[8,9]$.

\section{Les protocoles douleur sont indispensables. Sont-ils obligatoires ?}

Un protocole est essentiel aux urgences quand :

- l'urgence thérapeutique ne peut attendre la prescription nominative d'un médecin ;

- la présentation clinique d'un symptôme est subjective et les traitements simples et aspécifiques ;

- le domaine relève de compétences médicales transversales ;

Tableau 1 Les protocoles transposent l'état de l'art dans la pratique quotidienne

\begin{tabular}{|ll|}
\hline Recommandations & Protocole \\
\hline État de l'art & Concret/pratique \\
Nationales & Local ou locorégional \\
Générales & Adapté à une situation et/ou à \\
& un médicament \\
Par métier & Médical, médico-infirmier, \\
Par spécialité & de soins (personnel \\
& paramédical) \\
\hline
\end{tabular}






Fig. 1 Antalgie chez l'adulte en ventilation spontanée, recommandations formalisées d'experts 2010 (Vivien et al.) [7] : EVA : échelle visuelle analogique ; EN : échelle numérique ; EVS : échelle verbale simple ; MEOPA : mélange équimoléculaire oxygène protoxyde d'azote ; IV : intraveineux ; IVD : injection intraveineuse directe

- le niveau de formation pendant les études médicales est insuffisant pour homogénéiser les pratiques entre urgentistes ;

- le personnel infirmier a aussi un rôle propre et une marge de manœuvre à distance des médecins.

La prise en charge de la douleur traumatique est, par exemple, un bon champ d'application. Sans être obligatoire au sens strict, la mise en place de protocoles douleur dans les services d'accueil et d'urgences (SAU) est jugée indispensable dans le cadre des plans successifs de lutte contre la douleur [6] et préconisée par les recommandations d'experts : «L'évaluation et le traitement de la douleur doivent pouvoir être effectués par délégation par un personnel infirmier formé, dans le cadre d'un protocole de service connu de tous et sous réserve qu'un médecin urgentiste puisse intervenir sans délai et à tout moment » [7]. Les établissements de santé ont l'obligation, lors de la visite de certification de leur établissement, de répondre à la pratique exigible prioritaire PEP 12a concernant la prise en charge de la douleur [10]. Ainsi, tous les SAU doivent se doter de protocoles de soins dans le cadre de l'amélioration de la prise en charge de la douleur.

Qui doit rédiger les protocoles douleurs aux urgences : algologue et/ou urgentiste?

Les urgentistes sont les nouveaux spécialistes de la douleur aiguë, dans leur service et de plus en plus dans la littérature. Ils maîtrisent une prise en charge douleur "spécialisée » dans un contexte très spécifique. Ils doivent formaliser leurs pratiques et leurs connaissances, et cette mission extraclinique est essentielle à la professionnalisation et à la spécialisation du métier : rédiger les protocoles du service ne peut 
être délégué au seul algologue. L'urgentiste doit donc logiquement rester maître de ses pratiques, et ne peut se contenter de reprendre des propositions des commissions institutionnelles générales comme le CLUD (Comité de lutte contre la douleur), d'autant plus que les urgentistes n'y siègent pas toujours.

Par exemple, en traumatologie l'expertise de l'urgentiste ne se limite pas à l'aspect chirurgical, le protocole douleur étant la partie « expertise médicale » indispensable de cette spécialité.

\section{Quels sont les bénéfices d'un protocole douleur?}

La conception, la mise en place et la mise à jour d'un protocole dans un service d'urgence représentent du temps, de l'énergie et des coûts pour le responsable, les équipes et le service. Cependant, nous devons plutôt envisager un protocole sous l'angle des bénéfices qu'il va générer pour les différents intervenants. Premièrement, l'amélioration de la qualité des soins : la mise en place de protocoles dans un SAU est associée à une diminution des délais et à une amélioration de l'adhésion aux recommandations de bonne pratique et de la qualité de la prise en charge, dans la douleur comme dans d'autres domaines. Dans le sepsis sévère, la mise en place des protocoles fondés sur les recommandations internationales permet d'améliorer significativement le délai d'administration ou la pertinence de l'antibiothérapie initiale $[11,12]$. Valentian et al. [13] ont récemment étudié l'impact de la mise en place d'un protocole formalisé de demande anticipée de radiographies par l'infirmière organisatrice de l'accueil (IOA) dans les traumatismes de cheville avec un mécanisme supposé d'entorse. Les résultats montrent une diminution de $46 \%$ du délai d'attente avant la prise en charge médicale initiale et de $53 \%$ du délai total de prise en charge globale. Dans les cas de colique néphrétique, la mise en place d'un protocole standardisé a permis de réduire de $49 \%$ le délai d'accès à un antalgique $(72 \pm 63$ vs $37 \pm$ 42 minutes ; $p=0,003$ ) [14]. Un deuxième bénéfice est l'harmonisation des pratiques médicales : les protocoles contribuent à la diffusion et à la mise à jour des connaissances, qui ne sont pas la préoccupation exclusive des seuls « experts douleur » du service. En harmonisant les pratiques, on évite aussi les conflits de prescriptions entre praticien avec une meilleure continuité des soins et une cohérence médicale de service accrue, y compris pour les plus jeunes. Troisièmement, les protocoles, en plus d'harmoniser et de sécuriser les pratiques, doivent aussi un objectif de simplification de la prescription et des procédures. Ainsi, un urgentiste doit pouvoir aller à l'essentiel, il doit avoir à sa disposition, dans le cadre du protocole, un « kit» d'outils validés et mis en forme : arbre décisionnel, ordonnances types ou préremplies, courriers pour les correspondants, conseils pour le patient, etc. Par ailleurs, la mise en place d'un protocole contribue aussi à l'allégement des autres missions des cadres médicaux et paramédicaux : former les équipes, créer une dynamique de sensibilisation à la douleur de l'ensemble du personnel du service, évaluer le volume de médicaments nécessaires, réaliser une étude sur la douleur au SAU (évaluation des pratiques professionnelles, sujets de mémoires, etc.). Les protocoles permettent en outre de répondre aux plaintes des patients avec des arguments protocolaires (apport médicolégal). De plus, la conception d'un protocole est source de bénéfices pour l'expert : elle lui impose de formaliser et d'appliquer ses connaissances dans une démarche EBM (Evidence Based Medicine), contribuant ainsi à leur assimilation et à leur mise à jour régulière. Il met aussi en pratique certaines connaissances théoriques, et c'est cet aller-retour permanent qui fait justement de lui un expert. Enfin, le travail en commun sur l'élaboration d'un protocole cimente une équipe : l'expert en charge de la conception du protocole devra constituer un groupe pluriprofessionnel de travail et de réflexion, sur des critères d'expertise, d'implication dans la situation de soins et de motivation. Cette réflexion sera accompagnée par le personnel d'encadrement médical et paramédical de chaque service [6].

\section{Comment protocoliser la réalisation d'un protocole?}

Une fois la situation de soins à améliorer définie, la conception du protocole doit répondre à une méthodologie précise.

\section{Mettre en place des conditions favorables et rechercher l'adhésion}

La conception par une équipe pluridisciplinaire est essentielle pour identifier les problèmes et leur apporter des réponses pragmatiques et réalistes. Il ne faut jamais envisager de protocole douleur sans audit préalable des problématiques quotidiennes des infirmiers(ères) : leur adhésion en dépend. Cette adhésion des équipes est un facteur clé de la réussite d'un protocole et doit donc faire partie des éléments d'évaluation initiaux lors de la mise en place des procédures. Un protocole qui répond à une problématique concrète accélère la prise en charge, valorise le rôle de chacun, aura toutes les chances de remporter l'adhésion. Dans l'étude de Valentian et al., les deux tiers des infirmières interrogées constataient une amélioration de la prise en charge sans surcharge de travail, et $84 \%$ étaient favorables à la mise en place d'autres protocoles de ce type en traumatologie [13].

\section{Recueillir les données utiles}

L'expert devra recueillir des informations locales et/ou générales pour pouvoir élaborer son protocole, qui devront 
figurer sur le document écrit final dans un cadre réglementaire et légal précisé [15] : un état des lieux local du service, formalisé en une évaluation des pratiques professionnelles (insuffisance de prise en charge de telle ou telle pathologie ciblée, etc.). L'état de l'art scientifique pour le domaine concerné (guidelines, EBM, références scientifiques nationales et/ou internationales). Les textes légaux et réglementaires nationaux qui encadrent la mise en place de protocoles douleur et officialisent la délégation de soins au personnel infirmier (ou à d'autres professionnels paramédicaux tels que les manipulateurs radio) $[6,16]$, les protocoles de prise en charge initiale de la douleur étant considérés comme des prescriptions anticipées. Enfin, une liste de références doit figurer dans la fiche du protocole, par exemple dans un paragraphe intitulé « Cadre juridique » ou « Exigences à appliquer ». Dans le cadre de la douleur aiguë, une dizaine de références de ce type peuvent être identifiées systématiquement (Tableau 2).

\section{Élaborer les procédures de soins à standardiser}

L'élaboration d'un protocole repose sur une réflexion et des réponses communes à quelques questions portant sur les procédures de soins visées et leur cadre d'application. D'une façon générale, les réponses à ces questions constitueront la trame quasi systématique d'un protocole d'antalgie au SAU [15] (In : Recueil des actes du Congrès national de la douleur, Société d'étude et de traitement de la douleur, juin 2001, 203-13) : le cadre juridique : quels sont les textes réglementaires relatifs à la prise en charge de la douleur ? Les objectifs de la procédure : quel(s) type(s) de douleur et quelle(s) situation(s) de soin doivent être améliorés ? Les situations cliniques de mise en place du protocole : quelles

Tableau 2 Textes légaux et réglementaires encadrant un protocole douleur aux urgences Mise à jour : mars 2017

\begin{tabular}{l}
\hline Thème \\
\hline Obligation d'évaluer et de traiter la douleur \\
L'information de la personne malade \\
Rôle de l'infirmier — évaluation et traitement de la douleur \\
Rôle du manipulateur d'électroradiologie médicale \\
— surveillance et continuité des soins \\
Obligation de mise en place de protocoles de prise en charge \\
de la douleur
\end{tabular}

Méthodologie d'élaboration des protocoles et sources bibliographiques

\section{Références, textes officiels}

- Article L. 1110-5 du Code de la santé publique (loi nº 2002-303 du 4 mars 2002 relative aux droits des malades et à la qualité du système de santé - modifiée par loi n ${ }^{\circ}$ 2016-87 du 2 février 2016 - art. 1)

- Article L. 1112-4 du Code de la santé publique (modifié par ordonnance $n^{\circ} 2010-177$ du 23 février 2010 - art. 1)

- Code de la santé publique - article R4127-35.

- Code de la santé publique - article R4311-2 et suivants

- Code de la santé publique — article R4351-3

- Circulaire DGS/DH/DAS n ${ }^{\circ}$ 99/84 du 11 février 1999 relative à la mise en place de protocoles de prise en charge de la douleur aiguë par les équipes pluridisciplinaires médicales et soignantes dans les établissements de santé et institutions médicosociales

- Direction de l'hospitalisation et de l'organisation des soins - Guide d'orientation : organiser la lutte contre la douleur dans les établissements de santé. Paris : Direction de l'hospitalisation et de l'organisation des soins (éditeur), 2002, $33 \mathrm{p}$.

Outils d'évaluation de la douleur

- Agence nationale de sécurité du médicament et des produits de santé (ANSM). Prise en charge des douleurs de l'adulte modérées à intenses — Mise au point (actualisée : 04/08/2011)

Traitement médicamenteux de la douleur (et autres méthodes non • Vivien B, Adnet F, Bounes V, et al. Recommandations pharmacologiques) formalisées d'experts 2010 : sédation et analgésie en structure d'urgence (réactualisation de la conférence d'experts de la SFAR de 1999). Annales françaises de médecine d'urgence. 2011;1:5771

Prise en charge de la douleur aiguë dans les services d'urgence dès l'accueil
- Circulaire DGS/DH/DAS n ${ }^{\circ}$ 99/84 du 11 février 1999 relative à la mise en place de protocoles de prise en charge de la douleur aiguë par les équipes pluridisciplinaires médicales et soignantes dans les établissements de santé et institutions médicosociales 
sont les situations où l'infirmier(ère) pourra décider de l'application du protocole ? Quels sont les critères d'inclusion des patients? Les limites à l'utilisation du protocole : quelles sont les situations qui seront exclues de cette procédure ? Quels sont les critères d'exclusion des patients ? Le public concerné par ce protocole : qui administre et applique le protocole au sein du service ? Les outils d'évaluation : quels sont les outils d'évaluation de l'intensité de la douleur qui seront utilisés ? À quel moment précis se feront l'évaluation et la réévaluation? Les lieux géographiques : quels sont les lieux où ce protocole peut être mis en place (locaux...) ? Les agents antalgiques : quels sont les antalgiques utilisés dans ce protocole ? Par l'intermédiaire du protocole, les médecins et le chef de service autoriseront, selon des critères bien définis et sous leur responsabilité, l'infirmier(ère) à délivrer un produit médicamenteux.

\section{Rédiger le protocole « de référence »}

Le document écrit fera la synthèse des réponses aux questions posées précédemment. Il pourra donc suivre le plan suivant [15] : Domaine et limites d'application, descriptif des outils d'évaluation de l'intensité de la douleur, indications et contre-indications qui déclenchent l'éligibilité au protocole. Viennent ensuite la conduite à tenir infirmière ou démarche avant d'utiliser le protocole (la reconnaissance de la situation de soin, l'évaluation de la douleur, le choix selon l'arbre décisionnel du traitement à administrer, la recherche des contre-indications à l'utilisation du traitement, l'évaluation de l'efficacité et la surveillance du traitement, la retranscription de l'évaluation de l'intensité de la douleur) et enfin le rôle du médecin prescripteur (prescription initiale ou validation a posteriori, notamment).

\section{Créer et mettre en forme les documents pratiques}

Outre le document officiel, le protocole doit comprendre divers documents annexes pour optimiser sa diffusion et son appropriation, répondre à des exigences de traçabilité, ou fournir des outils concrets : fiche avec algorithme de soin, affichage pratique résumé, mémento de poche, outils d'évaluation, documents de traçabilité des actes effectués, conseils écrits, ordonnance de sortie... Si le fond est essentiel, la forme donnée au protocole et à ses annexes est également importante pour faciliter la lecture, la compréhension, l'affichage... L'expert en charge du protocole est ainsi en interaction avec plusieurs intervenants au cours de la conception, de la rédaction et de la validation du protocole (Fig. 2), c'est

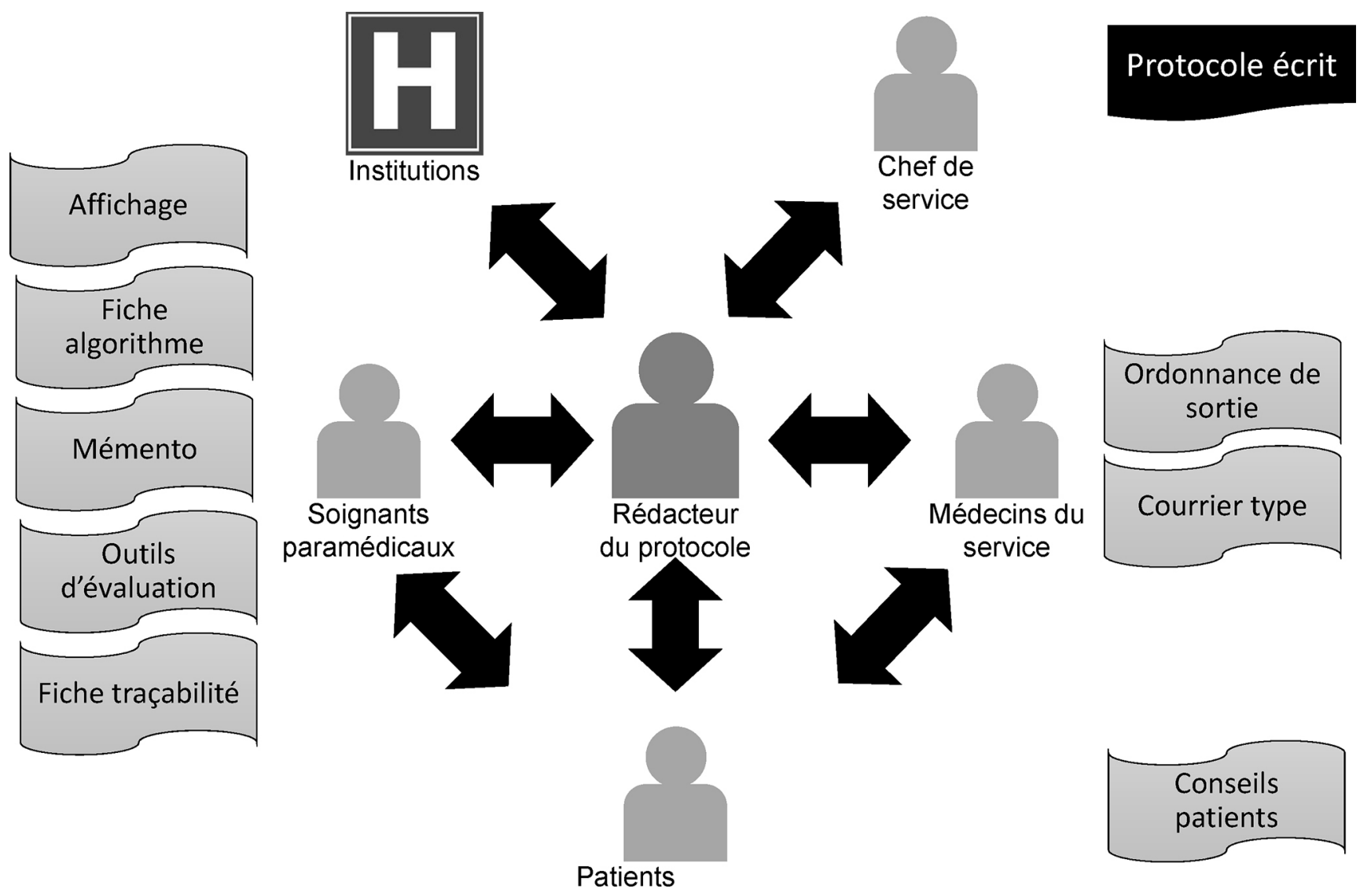

Fig. 2 Interfaces de l'expert rédacteur et documents pratiques dans le cadre d'un protocole 
ce que les documents doivent refléter. Ici encore, utiliser des modèles permet de gagner du temps. Des modèles de protocole existent, facilement accessibles et adaptables (au format PDF ou Word sur des sites hospitaliers institutionnels ou collaboratifs tels que www.urgences-serveur.fr).

\section{Faire valider le protocole}

Le protocole devra enfin être validé par l'ensemble de l'équipe médicale, par le pharmacien hospitalier ou pharmacien gérant de l'établissement et par le directeur du service des soins infirmiers, et obligatoirement daté et signé par le médecin responsable et le cadre de santé du service [6].

\section{Assurer la diffusion, l'évaluation et la mise à jour}

Les protocoles de soins doivent être $[6,15]$ :

- diffusés à l'ensemble du personnel médical et non médical du service, au directeur de l'établissement, au directeur du service des soins infirmiers et au pharmacien hospitalier;

- remis à chaque nouveau personnel dès son arrivée ;

- accessibles en permanence dans le service (référencés dans un classeur identifié et, selon l'organisation du service, affichés) ;

- évalués et si nécessaire réajustés et, dans ce cas, redatés et signés ;

- mis à jour obligatoirement au moins une fois par an ;
- revalidés systématiquement à chaque changement de l'un des signataires et rediffusés.

C'est pourquoi il faut que la première élaboration soit facilitée. Une expérience « traumatisante » empêche cette dernière étape d'aller à son terme, l'expert ne souhaitant plus réintervenir et assurer le suivi du protocole. Pourtant, une culture antidouleur ne peut s'instaurer dans un service sans continuité et amélioration par étapes. Un schéma résumant les différentes étapes est proposé dans la figure 3 [17]. Il permet à l'expert en charge du projet de ne pas perdre de temps à réinventer une méthodologie connue et incontournable.

\section{Conclusion}

La mise en place de protocoles antalgiques actualisés dans les SAU est indispensable pour l'optimisation des soins. Leur formalisme peut paraître contraignant et chronophage, et entraîner une inertie au changement. Mais nous devons également mesurer l'opportunité que ce travail représente en termes d'actualisation et de diffusion des connaissances, de formation, de cohésion des équipes et de démarche qualité. L'urgentiste doit s'approprier cette mission en tant que nouveau spécialiste de la douleur aiguë. L'optimisation de cette tâche et un gain de temps sont possibles en partant de bases existantes, de modèles, de documents types et de guides méthodologiques, permettant de consacrer son temps au

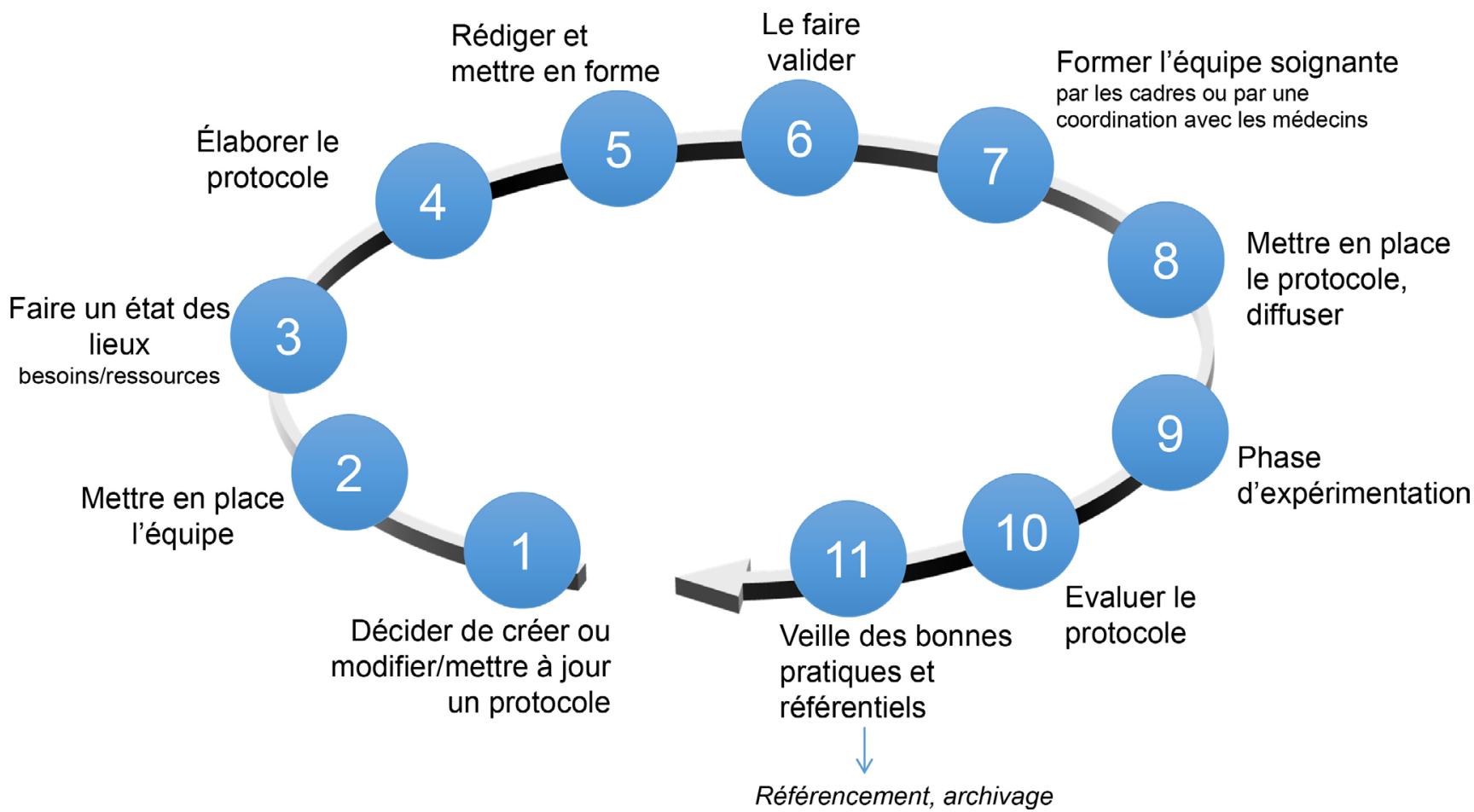

Fig. 3 Créer ou mettre à jour un protocole : étapes types 
contenu plutôt qu'à la forme. L'urgentiste est familier de l'utilisation de « kits » (kit d'intubation, etc.), nous pouvons proposer un kit d'outils et de procédures pour que cette mission extraclinique soit atraumatique. Culture institutionnelle et médecine d'urgence peuvent cohabiter sans douleur. En résumé, il est urgent d'élaborer des protocoles... pour rédiger nos protocoles.

Liens d'intérêt : V.-E. Lvovschi : activité de conseil pour Mundipharma. Mise à jour : janvier 2018.

\section{Références}

1. Lvovschi VE, Aubrun F (2014) Prise en charge de la douleur aux urgences : mise au point. J Eur Urg Reanim 4483:1-62

2. Ricard-Hibon A, Bounes V (2012) Sédation et analgésie en structure d'urgence. Quelles sédation et analgésie chez le patient en ventilation spontanée en structure d'urgence ? Ann Fr Anesth Reanim 31:295-312

3. Pickering G, Macian N, Libert F, et al (2014) Buccal acetaminophen provides fast analgesia: two randomized clinical trials in healthy volunteers. Drug Des Devel Ther 8:1621-7

4. Coffey F, Dissmann P, Mirza K, et al (2016) Methoxyflurane analgesia in adult patients in the emergency department: a subgroup analysis of a randomized, double-blind, placebocontrolled study (Stop!). Adv Ther 33:2012-31

5. Dictionnaire Larousse en ligne. Larousse Éd., Paris. http://www. larousse.fr/dictionnaires/francais/protocole/64577 (Dernier accès le 17 septembre 2017)

6. Direction générale de la santé, Bureau SQ 2, Direction des hôpitaux, Direction de l'action sociale (1999) Circulaire DGS/DH/ DAS n ${ }^{\circ}$ 99/84 du 11 février 1999 relative à la mise en place de protocole de prise en charge de la douleur aiguë par les équipes pluridisciplinaires médicales et soignantes dans les établissements de santé et institutions médico-sociales. http://social-sante.gouv. fr/fichiers/bo/1999/99-08/a0080531.htm

7. Vivien B, Adnet F, Bounes V, et al (2011) Recommandations formalisées d'experts 2010 : sédation et analgésie en structure d'ur- gence (réactualisation de la conférence d'experts de la SFAR de 1999). Ann Fr Med Urgence 1:57-71

8. Tourtier JP, Raynaud L, Murat I, et al (2010) Audit of protocols for treatment of paediatric burns in emergency departments in the Ile de France. Burns 36:1196-200

9. Schafer N, Driessen A, Frohlich M, et al (2015) Diversity in clinical management and protocols for the treatment of major bleeding trauma patients across European level I Trauma Centres. Scand J Trauma Resusc Emerg Med 23:74

10. Haute Autorité de santé. Direction de l'amélioration de la qualité et de la sécurité des soins (2014) Manuel de certification des établissements de santé V2010 - édition janvier 2014. https://www. has-sante.fr/portail/upload/docs/application/pdf/2014-03/ manuel_v2010_janvier2014.pdf

11. Francis M, Rich T, Williamson T, et al (2010) Effect of an emergency department sepsis protocol on time to antibiotics in severe sepsis. CJEM 12:303-10

12. Sweet DD, Jaswal D, Fu W, et al (2010) Effect of an emergency department sepsis protocol on the care of septic patients admitted to the intensive care unit. CJEM 12:414-20

13. Valentian M, Mewasing BI, Burggraff E, et al (2017) Intérêt d'une demande anticipée de radiographies par l'infirmière organisatrice de l'accueil, dans les traumatismes de cheville. Ann Fr Med Urgence 7:77-84

14. Steinberg PL, Nangia AK, Curtis K (2011) A standardized pain management protocol improves timeliness of analgesia among emergency department patients with renal colic. Qual Manag Health Care 20:30-6

15. Direction de l'hospitalisation et de l'organisation des soins (2002) Guide d'orientation : organiser la lutte contre la douleur dans les établissements de santé. Direction de l'hospitalisation et de l'organisation des soins (éditeur), Paris, 33 p. http://fulltext.bdsp. ehesp.fr/Ministere/Dhos/Publications/2002/guide_douleur.pdf

16. République française (2004) Code de la santé publique, article R4311-2 et suivants. Codifié par : décret 2004-802 du 29 juillet 2004. Version en vigueur au 8 août 2004. https://www.legifrance. gouv.fr/affichCodeArticle.do?cidTexte=LEGITEXT000006072665\&idArticle=LEGIARTI000006913889

17. Direction de l'hospitalisation et de l'organisation des soins. Circulaire $n^{\circ}$ DHOS/E2/2002/266 du 30 avril 2002, relative à la mise en œuvre du Programme national de lutte contre la douleur 2002-2005 dans les établissements de santé. NOR : MESH0230257C (texte non paru au Journal officiel) 